\title{
Open LabBot and RmsiGUI: Community development kit for sampling automation and ambient imaging
}

\author{
Ignacio Rosas-Román ${ }^{1,+}$, Cesaré Ovando-Vázquez ${ }^{2, \dagger}$, Abigail Moreno-Pedraza ${ }^{1}$, Héctor \\ Guillén-Alonso ${ }^{1}$ and Robert Winkler ${ }^{1, *(D)}$ \\ 1 Center for Research and Advanced Studies (CINVESTAV) Irapuato, Department of Biochemistry and \\ Biotechnology, Km. 9.6 Libramiento Norte Carr. Irapuato-León, 36824 Irapuato Gto., Mexico \\ 2 CONACYT Potosino Insitute of Scientific and Technological Research (IPYCIT), National Supercomputing \\ Center, Camino a la Presa San José 2055, Col. Lomas 4ta Sección, 78216 San Luis Potosí S.L.P., Mexico \\ * Correspondence: robert.winkler@cinvestav.mx; Tel.: +52 (462) 6239635 \\ + These authors contributed equally to this work.
}

Received: date; Accepted: date; Published: date

\begin{abstract}
Mass spectrometry research laboratories reported multiple probes for ambient ionization in the last years. Combining them with a mechanical moving stage enables automated sampling and imaging applications. We developed a robotic platform, which is based on RepRap 3D-printer components, and therefore easy to reproduce and to adopt for custom prototypes. The minimal step width of the Open LabBot is $12.5 \mu \mathrm{m}$, and the sampling dimensions $(x, y, z)$ are $18 \times 15 \times 20$ $\mathrm{cm}$. Adjustable rails in an aluminium frame construction facilitate the mounting of additional parts such as sensors, probes, or optical components. The Open LabBot uses industry-standard G-code for its control. The simple syntax facilitates the programming of the movement. We developed two programs: 1) LABI-Imaging, for direct control via a USB connection and the synchronization with MS data acquisition. 2) RmsiGUI, which integrates all steps of mass spectrometry imaging: The creation of G-code for robot control, the assembly of imzML files from raw data and the analysis of imzML files. We proved the functionality of the system by the automated sampling and classification of essential oils with a PlasmaChip probe. Further, we performed an ambient ionization mass spectrometry imaging (AIMSI) experiment of a lime slice with laser desorption low-temperature plasma (LD-LTP) ionization, demonstrating the integration of the complete workflow in RmsiGUI. The design of the Open LabBot and the software are released under open licenses to promote their use and adoption in the instrument developers' community.
\end{abstract}

Keywords: ambient ionization; mass spectrometry; high-throughput sampling; imaging; modular robot; open hardware; lab automation; peer production; open software; low-temperature plasma.)

\section{Introduction}

Ambient ionization mass spectrometry (AIMS) methods allow direct investigation of objects in their natural conditions [1]. The sample manipulation is minimized, resulting in simplified analytical workflows [2]. The study of compounds at ambient temperature and pressure also provides more relevant information about the 'real world' [3].

The enormous potential of AIMS is obvious. Thus, multiple AIMS methods were reported quickly after the introduction of the first methods [4], desorption electrospray ionization (DESI) [5], and direct analysis in real time (DART) [6]. In 2019, about fifty AIMS methods had been reported, with applications in direct chemical monitoring, forensics, bioanalytics, food control, among others [7].

Many AIMS techniques are suitable for screening the distribution of molecules on surfaces. The non-destructive analysis of large samples, such as works of art [8], is possible since the ionization does not require vacuum conditions. AIMS imaging (AIMSI) generates extremely information-rich data sets, combining the presence of molecules with their localization in a sample, such as biological tissue [9]. 
Translating novel ionization probes into practical applications still requires additional effort: 1) Mounting the probe onto a mass spectrometer with a defined geometry. 2) Moving the sample with precise positioning and timing, 3) for AIMSI, the combination of sampling positions and MS scans, and the analysis of result files. Building AIMSI prototypes is challenging and often requires considerable programming effort $[8,10,11]$, which hinders their adoption in other laboratories. Commercially available AIMS systems, on the other side, are usually not designed to facilitate the implementation of alternative analytical tools.

For building a generic AIMS robotics platform, we decided to use 3D-printer/ RepRap technology [12], because the components are readily available, of precise, and economical. The control programming language G-code [13] is simple to program and is supported a huge community. We already use 'fused deposition modeling' (FDM) to build reproducible low-temperature plasma (LTP) probes [14], and 3D-printing is becoming increasingly popular in the rapid prototyping of analytical instruments [15-20].

The integration of data handling is crucial for AIMSI applications. The imzML data format [21] has developed to the community standard in mass spectrometry imaging (MSI). For maintaining compatibility with other programs [22], we implemented the imzML format in our data processing software.

In this article, we describe the technical design of the Open LabBot. Further, we present the programs for robot control 'LABI-Imaging', and MSI data processing, 'RmsiGUI'. We demonstrate possible AIMS applications with two examples: 1) Automated sampling from vials with PlasmaChip ionization for the classification of essential oils, and 2) AIMSI of a lime slice with laser desorption low-temperature plasma (LD-LTP) ionization.

\section{Results and Discussion}

\subsection{Modular concept of the Open LabBot and G-code control}

The mechanical construction of the Open LabBot (Figure 1) facilitated the fast development of two different ambient ionization mass spectrometry (AIMS) systems; a high-throughput sampler and an AIMS imaging platform. We discuss the performance of both instrumental set-ups below.

With the currently used components, the Open LabBot allows automatic movements of $18 \times 15 \times$ $20 \mathrm{~cm}$ in $\mathrm{x}_{-}, \mathrm{y}$ - and $\mathrm{z}$-axis, respectively. The length of the ion transfer tube to the mass spectrometer limits the usable range for the y-axis to $7 \mathrm{~cm}$. The technical limit of single step movements is $12.5 \mu \mathrm{m}$. Altogether, the technical specifications of the RepRap motors meet the requirements for developing AIMS applications in our lab, which are mainly the high-throughput screening of food [24,25] and the biological mass spectrometry imaging (MSI) of macroscopic samples [10,23]. The open and modular design enables the adjustment of the Open LabBot to custom needs, such as particular sample dimensions or augmented lateral resolution.

Programming the robot movements is very easy (Figure 2). The following G-code is sufficient for moving the sample carrier from one sampling spot to another, with a pause of $1 \mathrm{~s}$ :

G21

G91

G4 S1.000000

G0 Y5.000000

G4 S1.000000

Table 1 lists the commands used in this example. The webpage http:/ / reprap.org/wiki/G-code informs more comprehensively about possible G-code operations. 


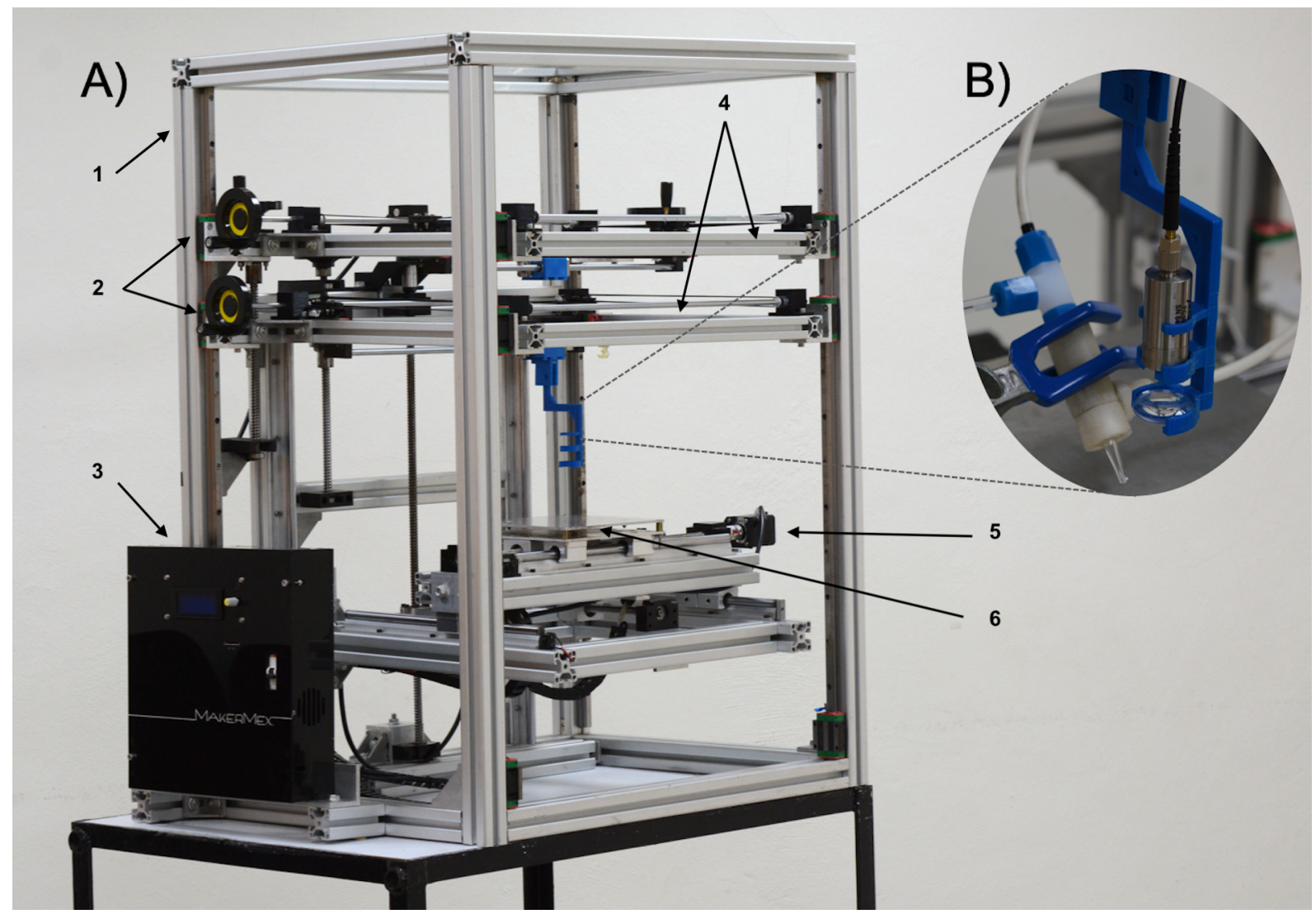

Figure 1. Technical design of the Open LabBot. (A) Overview: 1 - aluminium frame construction, 2 - rotating knobs for manual adjustment, 3 - Arduino control unit, 4 - rails for mounting objects, 5 stepper motor, 6 - sample table. (B) Example configuration: 3D-printed holder for a laser collimator and a lens for laser desorption (LD), and a 3D-LTP ionization probe [14,23].

A)

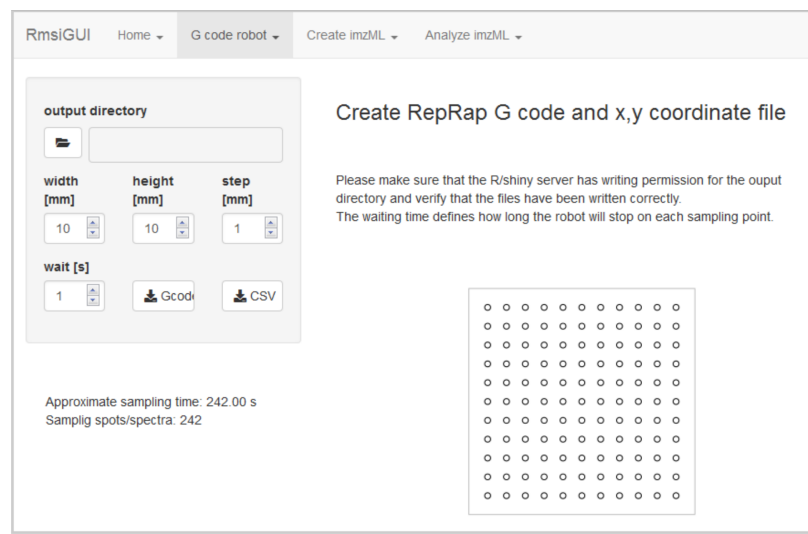

B)

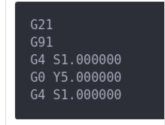

C)

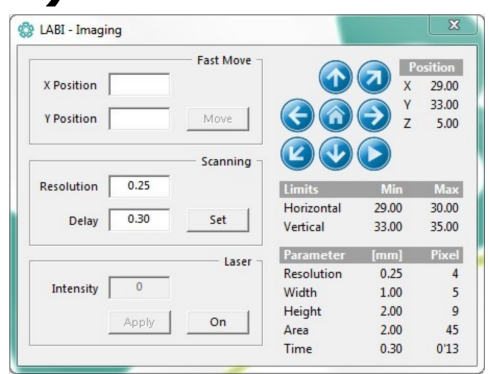

Figure 2. Options to control the Open LabBot: (A) RmsiGUI includes a module for G-Code file generation. (B) A G-Code text file with the extension .GCode, created by a custom Python script. The G-Code files of (A) and (B) are executed from a SD Card. (C) LABI-Imaging program, using direct communication via the USB port, to send G-Code commands to the Open LabBot and to synchronize with the Thermo Scientific MS software. 
Table 1. Basic G-code commands for controlling the Open LabBot.

\begin{tabular}{lll}
\hline G-code & operation & example \\
\hline G21 & set metric & G21 \\
G91 & set relative movements & \\
G0 & relative movement & G0 Y5.000000 = move $5 \mathrm{~mm}$ in y-axis \\
G4 & wait & G4 $\mathrm{S} 1.000000=$ wait $1 \mathrm{~s}$ \\
\hline
\end{tabular}

Industrial production uses the computer numerical control (CNC) standard RS274 [13] for forty years (see also DIN 66025/ ISO 6983). The simplicity of the syntax guarantees long-term readability of the G-code program files.

A text file with the extension .GCode is sufficient for the standalone operation of the Open LabBot. After inserting an SD card with the .GCode file to the corresponding slot of the control unit, the program is available to the robot. The Open LabBot control unit has a small display and selector button to chose and start G-code programs, which allows the creation of a library of standard sampling procedures.

Generic CNC programs such as the open 3D-printer software Pronterface/ Printrun (http:/ / www. pronterface.com) allow controlling the Open LabBot from a personal computer via a USB connection,

Initially, we created the G-code for sampling runs by simple R (https: / www.r-project.org/) or Python (https://www.python.org/) scripts. However, to facilitate the robot control by non-expert users, and to integrate additional functions, we developed a software application for ambient ionization mass spectrometry imaging (AIMSI): 'LABI-Imaging'.

\subsubsection{LABI-Imaging MSI software}

LABI-Imaging is able to perform several control tasks for mass spectrometry (MSI) acquisition: 1) Moving the Open LabBot platform by G-code commands, using a USB connection, 2) setting the laser power output level, 3) setting a mass spectrum filename, 4) starting/ stopping the mass scanning in the Thermo Scientific data acquisition software (LTQ Tune Plus Version 2.7.0.1103 SP1) and 5) monitoring the status of data acquisition (busy/end).

We implemented two MS data acquisition strategies:

1) One MS file per position

This operation mode saves exactly one MS file for each sampling position. The spectrum file name corresponds to the sampling position. The file handling and creation of imzML data sets are straightforward for this strategy. However, the procedure is relatively slow, $\sim 5 \mathrm{~s}$ per spectrum, due to limitations of the MS data acquisition software. Therefore, this strategy is mainly useful for automated sampling, e.g., of racks or microtiter plates. We used this strategy for the classification of essential oils with PlasmaChip ionization described below.

2) Continuous MS data acquisition

The continuous MS data acquisition strategy saves all micro scans in a single file. This method is approximately ten times faster, resulting in $~ 500 \mathrm{~ms}$ per spectrum, and therefore preferable for MSI. Since the MS files do not precisely match the sampling positions, assembling imzML files requires the interpolation of positioning data and spectral scans.

We provide the source code of LABI-Imaging under the terms GNU General Public License, version 3, (http://gplv3.fsf.org/) at https://bitbucket.org/lababi/open_labbot/.

\subsubsection{RmsiGUI}

RmsiGUI integrates the different data processing steps of a mass spectrometry imaging (MSI) experiment: 1) Creation of G-code (Figure 2A), 2) assembly of imzML files from mass spectra and position data, and 3) analysis of imzML files (Figure 4). The different functions are independent of each other. RmsiGUI is compatible with imzML files from on other platforms and compiled e.g., with the imzML Converter (https://www.cs.bham.ac.uk/ ibs/imzMLConverter/) [26]. 
We programmed RmsiGUI in the computational statistics and graphics language R (https:// www.r-project.org/) [27]. For processing mzML and imzML data, the program uses the libraries MALDIquant and MALDIquantForeign [28]. We used R/shiny (https:/ / shiny.rstudio.com/) to create as an interactive web application, which provides as a graphical user interface (GUI).

The organization of the GUI represents the MSI workflow:

G-code robot

In this tab, the user defines the sampling area and parameters. RmsiGUI creates the .GCode and sampling position CSV file that correspond to these settings. (Figure 2A).

Create imzML

The tab provides the creation of imzML files from mass spectra and position data, which result from different experimental MSI strategies:

- $\quad$ From a single mzML and a synchronization file, as produced by the LABI-Imaging software in the 'continuous MS data acquisition' mode.

- From a directory containing mzMLs and image width in pixels. The function assumes an

S-shaped sampling path, corresponding to the LABI-Imaging software, with the 'one MS file per position' strategy.

- $\quad$ From a single mzML and an x,y CSV file, as exported from the RmsiGUI G-code robot tab.

Analyze imzML

The imzML files can be loaded and visualized in the last tab. RmsiGUI provides various options to represent the signal intensities, such as simple pixel maps, contour lines, and overlays with sample photos. Both profile and centroid MS data are supported. The spectrum viewer either shows the sum MS spectrum or the MS spectrum of a single pixel, selected by a crosshair (Figure 4).

The representation of signal intensities in mass images by rainbow colors or other sub-optimal color maps can be misleading and give a false impression on the distribution of a molecule [29]. For a correct representation of intensity levels, we, therefore, use the viridis color map, developed by Jamie R. Nuñez and Sean M. Colby (https:/ / cran.r-project.org/package=viridis).

The source code is available from https://bitbucket.org/lababi/rmsigui/, under the terms of the GNU General Public License, version 3 (http:/ / gplv3.fsf.org/). The following lines demonstrate the installing and running of RmsiGUI from the R command line or RStudio (https:/ / www.rstudio.com/):

Install $\mathrm{R}$ package from BitBucket:

library (devtools)

install_bitbucket ('lababi/rmsigui')

Run RmsiGUI by:

library (RmsigUI)

launch()

RmsiGUI opens in the default web browser.

\subsection{Classification of essential oils with a PlasmaChip}

The PlasmaChip is a microwave-driven plasma ionization source, which was recently introduced by NovionX GmbH (Germany, http:/ / www.novionx.de). For sampling the headspace of essential oil samples, we mounted the probe perpendicularly to the extended ion inlet tube of the mass spectrometer, using a 3D-printed adapter (Figure 3A). We used the LABI-Imaging program to move the sample table according to the geometry of the sample rack and to record one MS spectrum for each sample. Analyzing the mzML spectra in R, applying principal component analysis (PCA) and hierarchical clustering analysis (HCA), resulting in the correct grouping of the samples (Figure 3B and 3C).

This experiment demonstrates the simplicity to develop an analytical screening platform featuring a custom probe, using the Open LabBot. 
A)

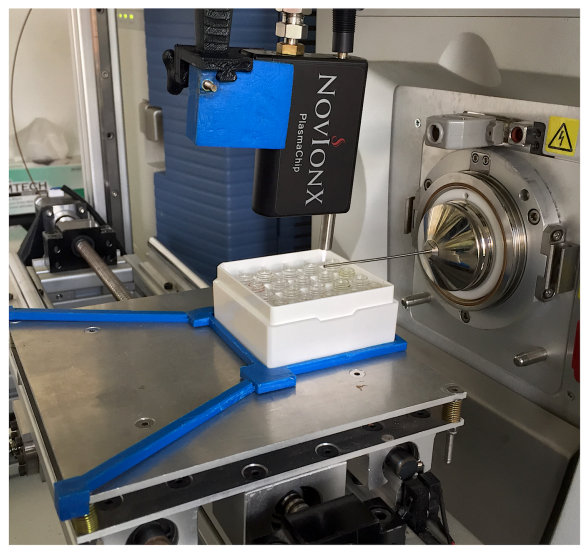

B)

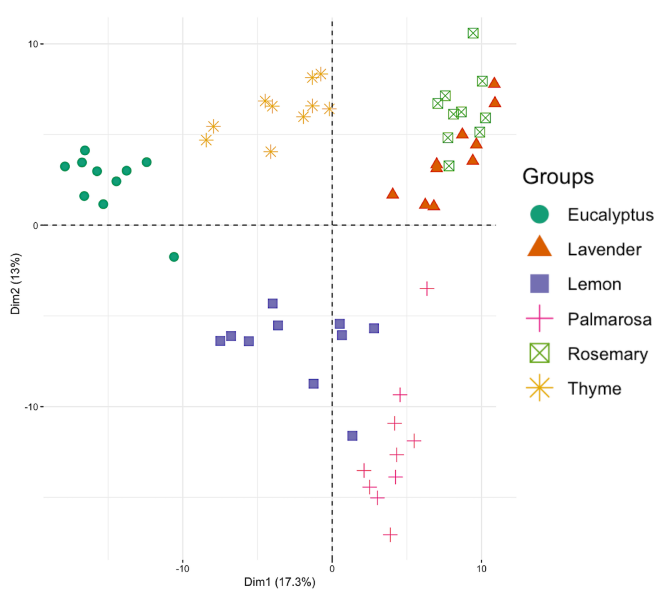

C)

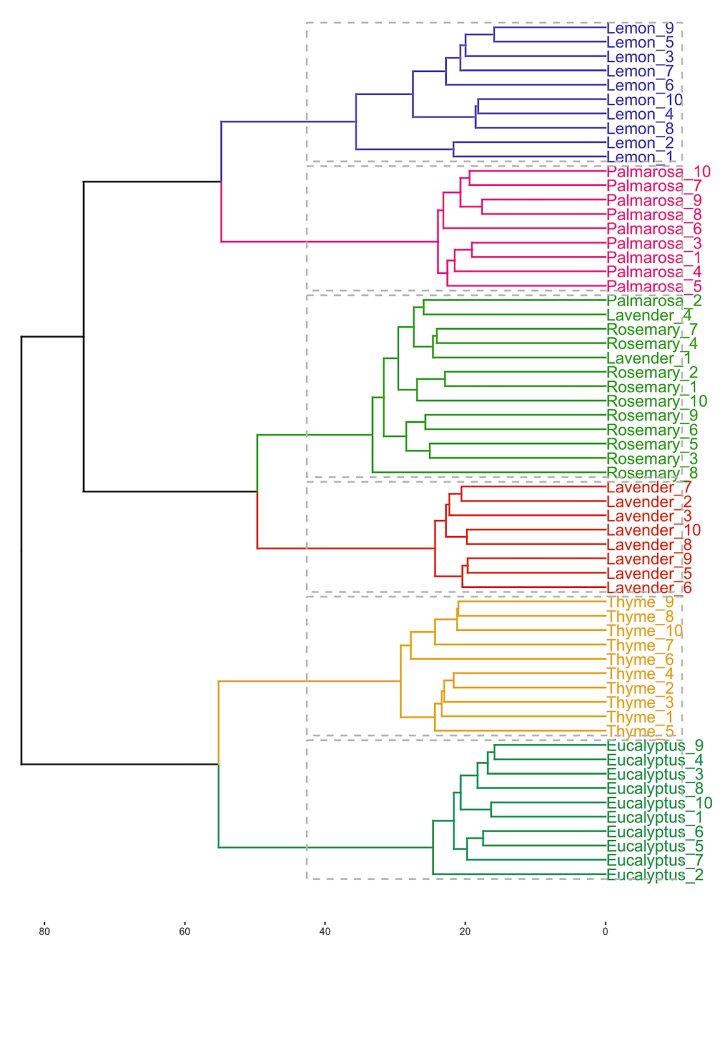

Figure 3. Analysis of essential oils with PlasmaChip ionization. (A) PlasmaChip mounted at $90^{\circ}$ relatively to the sampling platform, using 3D-printed parts to adapt the ion source and the sample rack. (B) Principal component analysis (PCA) and (C) a dendrogram, based on a hierarchical clustering analysis (HCA) from the acquired mass spectrometry data, lead to the correct classification of the samples. 

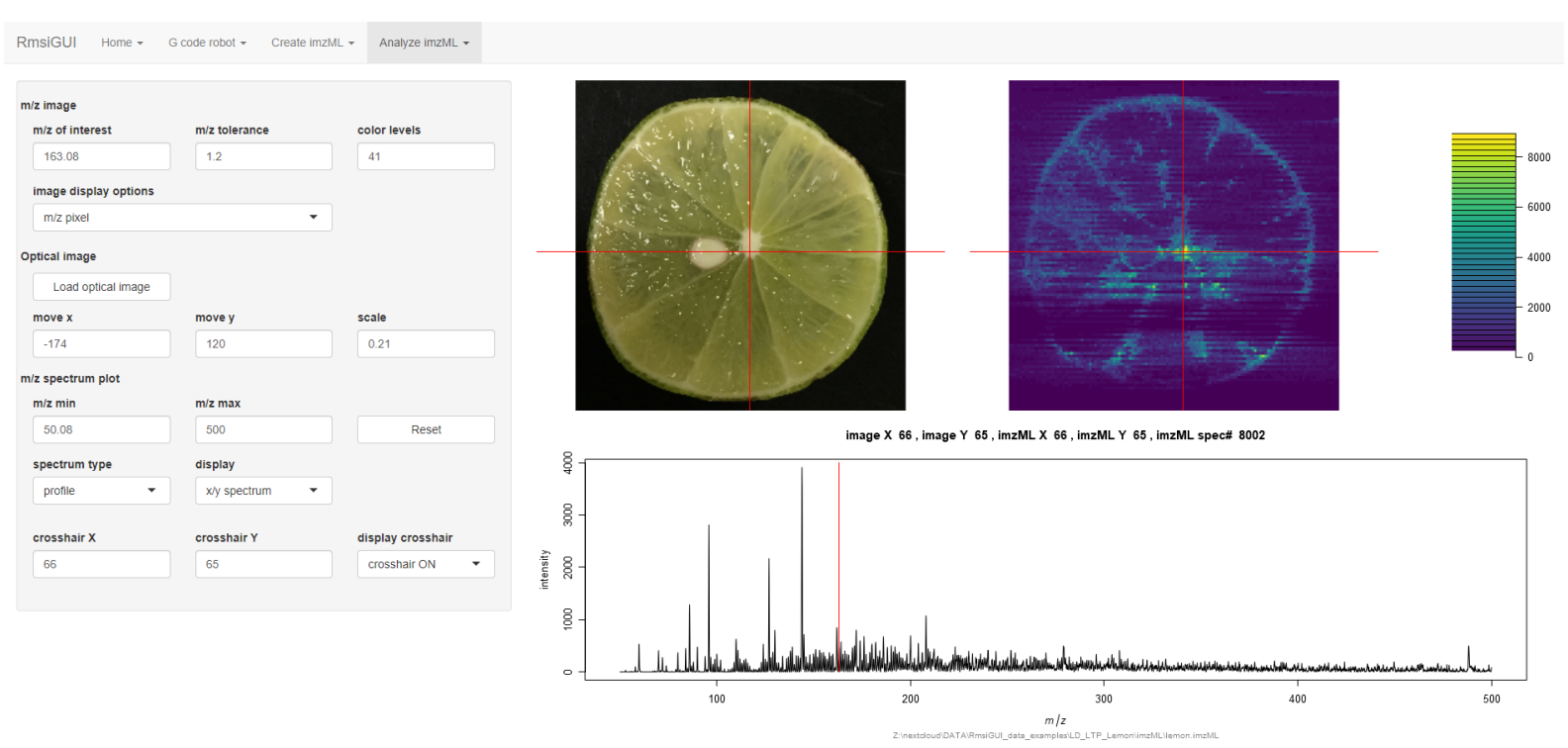

Figure 4. Mass spectrometry imaging (MSI) of a Mexican lime (Citrus $\times$ aurantiifolia) slice, using the Open LabBot with laser desorption/ low-temperature plasma ionization. The results are analyzed and visualized with RmsiGUI: The red crosshair for selecting single spectra is synchronized between the sample photography and the mass intensity image. Either the master mass spectrum of the entire imzML file or the mass spectrum of the selected pixel is displayed at the bottom; the mass range and data mode (centroid/ profile) can be adjusted on the left panel. For the visualization of mass intensity maps, the $m / z$ value and its tolerance for integration can be chosen manually at the top. RmsiGUI allows the loading and moving/ zooming of the sample image, as well as different visualization modes.

\subsection{Ambient Ionization Mass Spectrometry Imaging (AIMSI)}

The development of ambient ionization mass spectrometry imaging (AIMSI) systems is challenging because it requires the coupling and synchronous control of various components. Additionally, an efficient data processing pipeline is necessary to compile result files from the raw data correctly. Based on the Open LabBot, we built an AIMSI system, which uses a laser for the desorption (LD) of compounds and a 3D-printed low-temperature plasma (LTP) probe for their post-ionization.

With this LD-LTP MSI system, we studied an untreated lime slice. Our software LABI-Imaging controlled the laser, the sample movements, and the MS data acquisition. From the data, which were generated by LABI-Imaging and the MS, we built an imzML file using RmsiGUI and analyzed the imzML file in the same program.

The visualization of ion maps and spectra from the experiment with RmsiGUI (Figure 4) shows the successful integration of hardware and software, and the suitability of the system for AIMSI prototyping.

\section{Materials and Methods}

\subsection{Open LabBot}

The Open LabBot (Figure 1) consists of an aluminum frame construction with a sample table and movable rails for the mounting of diverse devices. The sample platform is driven by three stepper motors (Motech motor Co. Ltd., China), which allow movements in $\mathrm{x}, \mathrm{y}$ and $\mathrm{z}$ axes. The robot is controlled by a RUMBA Taurino with ATmega 2560 microprocessor. The sample table consists of an aluminum plate of $3 \mathrm{~mm}$ thickness and $18 \mathrm{~cm}$ in length and $18 \mathrm{~cm}$ in width. With the current set-up, movements of $18 \times 15 \times 20 \mathrm{~cm}$ in $\mathrm{x}^{-}, \mathrm{y}^{-}$, and $\mathrm{z}$-axis, respectively, are possible, with a lateral precision of $12.5 \mu \mathrm{m}$. 
Devices, such as sensors, optical components and probes, can be mounted on the rails which are placed above the sampling platform. These rails can be adjusted manually. The polymer parts shown in this article (holders, adapters etc.) were 3D-printed with acrylonitrile-butadiene-styrene (ABS) or polylactic acid (PLA). However, also other materials, such as polyethylene terephthalate (PET) or polycarbonate $(\mathrm{PC})$ are available for the 3D-printing of custom parts.

The Open LabBot supports the RS274 CNC command standard [13], commonly known as 'G-code language'. G-code files are plain text (http://reprap.org/wiki/G-code) allowing the generation of custom G-Code files with high-level programming languages such as python or R.

The Open LabBot was developed in collaboration with the 3D-printer company MakerMex (León, Mexico) and can be purchased as a kit or assembled from KUTURABI SA de CV (Irapuato, Mexico, http://kuturabi.com/). We release the design of the Open LabBot under the terms of the Creative Commons Attribution-NonCommercial 4.0 International Public License (http:/ / creativecommons. org/licenses/by-nc/4.0/legalcode), to encourage its reproduction, optimization and modification. However, to recover development costs and to support further projects, we ask commercial users to contact us for a technology transfer agreement.

\subsection{Software}

The Open LabBot can be controlled by a simple G-code file that is executed directly from a SD card, which can be inserted at the control unit. However, to facilitate the creation of control programs, and to integrate the downstream data processing, we developed two additional programs: 1) LABI-Imaging is a $\mathrm{C}++$ program for mass spectrometry imaging (MSI) data acquisition (synchonization of robot movements and MS data acquisition, control via the USB port). 2) RmsiGUI provides a graphical user interface for the creation of G-code, the assembly, and analysis of MSI data files in imzML data format. The different options to control the Open LabBot are displayed in Figure 2. The programs LABI-Imaging and RmsiGUI are described in more detail above (Results and Discussion).

\subsection{Analysis of essential oils by automated sampling with PlasmaChip ionization}

For the high-throughput analysis of essential oils (eucalyptus, lavender, lemon, palmarosa, rosemary and thyme (Just AG, Switzerland) we attached a PlasmaChip (NovionX GmbH, Germany, http: / / novionx.de) with a 3D-printed arm and placed it perpendicularly to the mass spectrometer extended ion inlet cone (Figure 3A). $50 \mu \mathrm{L}$ of each essential oil sample were filled into glass vials and placed randomly inside a rack. The rack was mounted on the Open LabBot with a 3D-printed adapter. The geometrical parameters were reported before [25]. The argon flow was set to $1.0 \mathrm{~L} / \mathrm{min}$ and regulated using a gas flow controller (Swagelok, Mexico). The LCQ Fleet mass spectrometer was set on positive mode, with a mass range of $50-500 \mathrm{~m} / \mathrm{z}$. The movement of the sample platform was controlled with the LABI-Imaging program. Each sample was analyzed ten times, changing the order of the vials. For reducing carry-over, the ion transfer was flushed by measuring a vial with pure ethanol after each sample. Raw data files were converted to .mzML with ProteoWizard [30], and analyzed using the statistics program R (http:/ / r-project.org/) [27] with the packages MALDIquant [28] and factoextra [31].

\subsection{Mass spectrometry imaging with laser desorption low-temperature plasma ionization}

For mass spectrometry imaging (MSI), we used a laser desorption low-temperature plasma ionization (LD-LTP) system developed in our laboratory [23]. The laser energy was controlled with an Arduino Uno board and a custom program, which can be downloaded from https: / / bitbucket.org/ lababi/open_labbot/ (licensed under GPL, V3), and set to $224 \mathrm{MW} / \mathrm{cm}^{2}$. The low-temperature plasma (LTP) probe was operated with a helium flow of $0.1 \mathrm{~L} / \mathrm{min}, 3 \mathrm{kV}$ output voltage and $12 \mathrm{kHz}$ frequency. The LCQ-Fleet ion trap mass analyzer (Thermo Scientific, USA.) was operated in the positive mode, with a mass range of 50-500 $\mathrm{m} / \mathrm{z}$. The spectra were acquired in full scan mode. The capillary voltage was set to $55 \mathrm{~V}$, the capillary temperature to $200^{\circ} \mathrm{C}$, and the tube lens voltage to $25 \mathrm{~V}$. The maximum 
time for ion trap injection was set to $300 \mathrm{~ms}$, with 2 microscans per spectrum, using the automatic gain control (AGC). The data set of Mexican lime (Citrus $\times$ aurantiifolia) was generated for a sampling area of $37.2 \times 37.2 \mathrm{~mm}$ with a step width of $300 \mu \mathrm{m}$ and a waiting time of $4 \mathrm{~s}$ for each spot. Raw mass spectrometry data files were converted to the mzML community format using ProteoWizard [30]. We used RmsiGUI to convert the mzML mass spectral data and $t x t(x, y)$ localization files to the imzML format, and to analyze and visualize the MSI data.

\section{Conclusions}

The Open LabBot provides a modular framework for the rapid prototyping of ambient ionization mass spectrometry (AIMS) systems. We demonstrated the versatility of the platform by assembling two distinct AIMS set-ups with plasma ionization: An automated sampling system and an AIMS imaging system.

For providing interested researchers the opportunity to re-use and re-mix the modules, we disclosed the hardware design and software with permissive licenses (Creative Commons and GPL). Additionally, we implemented industry (G-code CNC) and community standards (mzML, imzML), to simplify the compatibility with other systems and software solutions.

Author Contributions: Conceptualization, Robert Winkler; Formal analysis, Abigail Moreno-Pedraza, Héctor Guillén-Alonso and Robert Winkler; Funding acquisition, Robert Winkler; Investigation, Ignacio Rosas-Román, Cesaré Ovando-Vázquez, Abigail Moreno-Pedraza and Héctor Guillén-Alonso; Methodology, Ignacio Rosas-Román, Cesaré Ovando-Vázquez, Abigail Moreno-Pedraza and Héctor Guillén-Alonso; Project administration, Robert Winkler; Resources, Robert Winkler; Software, Ignacio Rosas-Román, Cesaré Ovando-Vázquez and Robert Winkler; Supervision, Robert Winkler; Writing - original draft, Ignacio Rosas-Román and Cesaré Ovando-Vázquez; Writing - review \& editing, Robert Winkler.

Funding: The project was funded by the CONACyT Fronteras project 2015-2/814 and the bilateral grant CONACyT-DFG 2016/277850. AMP and HGA acknowledge their CONACyT scholarships and COV his CONACyT Cátedra project 809.

Acknowledgments: We thank José Antonio Cisneros Durán for taking professional photos, and Dr. Maria Teresa Carrillo Rayas, Maria Isabel Cristina Elizarraraz Anaya, as well as Thermo Mexico for excellent technical support.

Conflicts of Interest: The authors declare the following competing financial interest(s): RW is co-inventor of the patent application "Non-thermal plasma jet device as source of spatial ionization for ambient mass spectrometry and method of application" (WO 2014/057409). RW is a shareholder of the company KUTURABI SA de CV that commercializes the Open LabBot.

\section{References}

1. Domin, M.; Cody, R. Ambient Ionization Mass Spectrometry; Royal Society of Chemistry, 2014.

2. Venter, A.; Nefliu, M.; Graham Cooks, R. Ambient desorption ionization mass spectrometry. TrAC Trends in Analytical Chemistry 2008, 27, 284-290. doi:10.1016/j.trac.2008.01.010.

3. Alberici, R.M.; Simas, R.C.; Sanvido, G.B.; Romão, W.; Lalli, P.M.; Benassi, M.; Cunha, I.B.S.; Eberlin, M.N. Ambient mass spectrometry: bringing MS into the "real world". Anal Bioanal Chem 2010, 398, 265-294. doi:10.1007/s00216-010-3808-3.

4. Chen, H.; Gamez, G.; Zenobi, R. What can we learn from ambient ionization techniques? Journal of the American Society for Mass Spectrometry 2009, 20, 1947-1963. doi:10.1016/j.jasms.2009.07.025.

5. Takáts, Z.; Wiseman, J.M.; Gologan, B.; Cooks, R.G. Mass Spectrometry Sampling Under Ambient Conditions with Desorption Electrospray Ionization. Science 2004, 306, 471-473. doi:10.1126/science.1104404.

6. Cody, R.B.; Laramée, J.A.; Durst, H.D. Versatile new ion source for the analysis of materials in open air under ambient conditions. Anal. Chem. 2005, 77, 2297-2302. doi:10.1021/ac050162j.

7. Feider, C.L.; Krieger, A.; DeHoog, R.J.; Eberlin, L.S. Ambient Ionization Mass Spectrometry: Recent Developments and Applications. Anal. Chem. 2019, 91, 4266-4290. doi:10.1021/acs.analchem.9b00807.

8. Liu, Y.; Ma, X.; Lin, Z.; He, M.; Han, G.; Yang, C.; Xing, Z.; Zhang, S.; Zhang, X. Imaging mass spectrometry with a low-temperature plasma probe for the analysis of works of art. Angew. Chem. Int. Ed. Engl. 2010, 49, 4435-4437. 00100, doi:10.1002/anie.200906975. 
9. Wu, C.; Dill, A.L.; Eberlin, L.S.; Cooks, R.G.; Ifa, D.R. Mass spectrometry imaging under ambient conditions. Mass Spectrom. Rev. 2013, 32, 218-243. doi:10.1002/mas.21360.

10. Maldonado-Torres, M.; López-Hernández, J.F.; Jiménez-Sandoval, P.; Winkler, R. 'Plug and Play' assembly of a low-temperature plasma ionization mass spectrometry imaging (LTP-MSI) system. Journal of proteomics 2014, 102C, 60-65. doi:10.1016/j.jprot.2014.03.003.

11. Bartels, B.; Kulkarni, P.; Danz, N.; Böcker, S.; Saluz, H.P.; Svatoš, A. Mapping metabolites from rough terrain: laser ablation electrospray ionization on non-flat samples. RSC Adv. 2017, 7, 9045-9050. doi:10.1039/C6RA26854D.

12. Jones, R.; Haufe, P.; Sells, E.; Iravani, P.; Olliver, V.; Palmer, C.; Bowyer, A. RepRap - the replicating rapid prototyper. Robotica 2011, 29, 177-191. doi:10.1017/S026357471000069X.

13. EIA Standard RS-274-D Interchangeable Variable Block Data Format for Positioning, Contouring, and Contouring/Positioning Numerically Controlled Machines; Electronic Industries Association: 2001 Eye Street, NW, Washington, D.C. 20006, 1979.

14. Martínez-Jarquín, S.; Moreno-Pedraza, A.; Guillén-Alonso, H.; Winkler, R. Template for 3D Printing a Low-Temperature Plasma Probe. Anal. Chem. 2016, 88, 6976-6980. bibtex: martinez-jarquin_template_2016, doi:10.1021/acs.analchem.6b01019.

15. Pearce, J.M. Laboratory equipment: Cut costs with open-source hardware. Nature 2014, 505, 618-618. doi:10.1038/505618d.

16. Zhang, C.; Wijnen, B.; Pearce, J.M. Open-Source 3-D Platform for Low-Cost Scientific Instrument Ecosystem. J Lab Autom. 2016, 21, 517-525. doi:10.1177/2211068215624406.

17. Tycova, A.; Prikryl, J.; Foret, F. Reproducible preparation of nanospray tips for capillary electrophoresis coupled to mass spectrometry using 3D printed grinding device. ELECTROPHORESIS 2016, 37, 924-930. doi:10.1002/elps.201500467.

18. Duarte, L.C.; Carvalho, T.C.d.; Lobo-Júnior, E.O.; Abdelnur, P.V.; Vaz, B.G.; Coltro, W.K.T. 3D printing of microfluidic devices for paper-assisted direct spray ionization mass spectrometry. Anal. Methods 2016, 8, 496-503. doi:10.1039/C5AY03074A.

19. Gross, B.; Lockwood, S.Y.; Spence, D.M. Recent Advances in Analytical Chemistry by 3D Printing. ANALYTICAL CHEMISTRY 2017, 89, 57-70. doi:10.1021/acs.analchem.6b04344.

20. Hollerbach, A.; Fedick, P.W.; Cooks, R.G. Ion Mobility-Mass Spectrometry Using a Dual-Gated 3D Printed Ion Mobility Spectrometer. Anal. Chem. 2018, 90, 13265-13272. doi:10.1021/acs.analchem.8b02209.

21. Römpp, A.; Schramm, T.; Hester, A.; Klinkert, I.; Both, J.P.; Heeren, R.M.A.; Stöckli, M.; Spengler, B. imzML: Imaging Mass Spectrometry Markup Language: A common data format for mass spectrometry imaging. Methods Mol. Biol. 2011, 696, 205-224. doi:10.1007/978-1-60761-987-1_12.

22. Weiskirchen, R.; Weiskirchen, S.; Kim, P.; Winkler, R. Software solutions for evaluation and visualization of laser ablation inductively coupled plasma mass spectrometry imaging (LA-ICP-MSI) data: a short overview. Journal of Cheminformatics 2019, 11, 16. doi:10.1186/s13321-019-0338-7.

23. Moreno-Pedraza, A.; Rosas-Román, I.; Garcia-Rojas, N.S.; Guillén-Alonso, H.; Ovando-Vázquez, C.; Díaz-Ramírez, D.; Cuevas-Contreras, J.; Vergara, F.; Marsch-Martínez, N.; Molina-Torres, J.; Winkler, R. Elucidating the Distribution of Plant Metabolites from Native Tissues with Laser Desorption Low-Temperature Plasma Mass Spectrometry Imaging. Anal. Chem. 2019, 91, 2734-2743. doi:10.1021/acs.analchem.8b04406.

24. Gamboa-Becerra, R.; Montero-Vargas, J.M.; Martínez-Jarquín, S.; Gálvez-Ponce, E.; Moreno-Pedraza, A.; Winkler, R. Rapid Classification of Coffee Products by Data Mining Models from Direct Electrospray and Plasma-Based Mass Spectrometry Analyses. Food Anal. Methods 2017, 10, 1359-1368. doi:10.1007/s12161-016-0696-y.

25. Martínez-Jarquín, S.; Moreno-Pedraza, A.; Cázarez-García, D.; Winkler, R. Automated chemical fingerprinting of Mexican spirits derived from Agave (tequila and mezcal) using direct-injection electrospray ionisation (DIESI) and low-temperature plasma (LTP) mass spectrometry. Analytical Methods 2017, 9, 5023-5028. doi:10.1039/C7AY00793K.

26. Race, A.M.; Styles, I.B.; Bunch, J. Inclusive sharing of mass spectrometry imaging data requires a converter for all. J Proteomics 2012, 75, 5111-5112. doi:10.1016/j.jprot.2012.05.035.

27. $\quad \mathrm{R}$ Core Team. R: A Language and Environment for Statistical Computing; R Foundation for Statistical Computing: Vienna, Austria, 2018. 
28. Gibb, S.; Strimmer, K. MALDIquant: a versatile R package for the analysis of mass spectrometry data. Bioinformatics 2012, 28, 2270-2271. doi:10.1093/bioinformatics/bts447.

29. Race, A.M.; Bunch, J. Optimisation of colour schemes to accurately display mass spectrometry imaging data based on human colour perception. Anal Bioanal Chem 2015, pp. 1-8. doi:10.1007/s00216-014-8404-5.

30. Kessner, D.; Chambers, M.; Burke, R.; Agus, D.; Mallick, P. ProteoWizard: open source software for rapid proteomics tools development. Bioinformatics 2008, 24, 2534-2536. doi:10.1093/bioinformatics/btn323.

31. Kassambara, A.; Mundt, F. factoextra: Extract and Visualize the Results of Multivariate Data Analyses; 2017. 